\title{
Myosin VI contributes to synaptic transmission and development at the Drosophila neuromuscular junction
}

\author{
Marta Kisiel, Debolina Majumdar, Shelagh Campbell and Bryan A Stewart
}

\begin{abstract}
Background: Myosin VI, encoded by jaguar (jar) in Drosophila melanogaster, is a unique member of the myosin superfamily of actin-based motor proteins. Myosin $\mathrm{VI}$ is the only myosin known to move towards the minus or pointed ends of actin filaments. Although Myosin VI has been implicated in numerous cellular processes as both an anchor and a transporter, little is known about the role of Myosin VI in the nervous system. We previously recovered jar in a screen for genes that modify neuromuscular junction (NMJ) development and here we report on the genetic analysis of Myosin VI in synaptic development and function using loss of function jar alleles.

Results: Our experiments on Drosophila third instar larvae revealed decreased locomotor activity, a decrease in NMJ length, a reduction in synaptic bouton number, and altered synaptic vesicle localization in jar mutants. Furthermore, our studies of synaptic transmission revealed alterations in both basal synaptic transmission and short-term plasticity at the jar mutant neuromuscular synapse.

Conclusions: Altogether these findings indicate that Myosin VI is important for proper synaptic function and morphology. Myosin VI may be functioning as an anchor to tether vesicles to the bouton periphery and, thereby, participating in the regulation of synaptic vesicle mobilization during synaptic transmission.
\end{abstract}

\section{Background}

While many of the molecular mechanisms that regulate the synaptic vesicle cycle are becoming understood, the methods by which vesicles move within the nerve terminal are less well known. Even though vesicle movement is an underlying assumption of most models of synaptic vesicle trafficking, it has only been demonstrated recently that the pool of vesicles is dynamic [1-3]. Understanding the mechanisms by which vesicles move within nerve terminals will inform our knowledge of both the exoand endocytic branches of the vesicle cycle.

Two modes of transport can be envisioned: diffusion and active transport. Some studies, such as an analysis of vesicles in the recycling pool, are consistent with the notion that simple diffusion is a contributing force [4]. However, several studies indicate that a myosin-based active transport mechanism may be important $[2,5,6]$, although there are some results to the contrary [7].

\footnotetext{
* Correspondence: bryan.stewart@utoronto.ca

Department of Biology, University of Toronto Mississauga, 3359 Mississauga Rd, Mississauga, ON, L5L 1C6, Canada
}

Members of the myosin-family of motor proteins share the feature of converting energy derived from ATP into molecular motion and they are used in a host of cellular processes including cell motility, cytokinesis, intracellular transport and muscle contraction.

Within the myosin superfamily of proteins, Myosin VI shares the well-conserved basic structure of other myosin proteins, however, it is the only myosin known to move towards the pointed (minus) ends of actin filaments. Myosin VI contains a unique insert present between the converter and the IQ motif of the motor domain that repositions its lever arm resulting in reverse directionality [8]. Additionally, another unique insert near the nucleotide-binding pocket of the motor domain regulates Myosin VI kinetics by restricting the accessibility of ATP to the nucleotide-binding pocket, which slows ADP release and hence, slows the dissociation of Myosin VI from actin [8]. The slow kinetic properties of Myosin VI suggest that it may function as an anchor that can link components to the actin cytoskeleton, in addition to functioning as a cargo transporter [9-12]. Myosin VI has been specifically 
implicated in a number of diverse cellular roles including stereocilia maintenance [13], spermatid individualization [14-16], border cell migration [17,18], nuclear transcription [19], epithelial cell-cell contacts [20,21], and regulating Golgi morphology and exocytosis [22,23]. In addition, Myosin VI is thought to have a function in the vesicle cycle in clathrin-mediated endocytosis since some splice variants of Myosin VI in human and mammalian tissues localize to clathrin-coated pits/vesicles [24]. In mammalian cells, Myosin VI has been shown to associate with endocytic vesicles following clathrin uncoating and to subsequently transport these uncoated vesicles through the actin-rich periphery to the early endosome [25]. Cells of Myosin VI knock-out mice exhibit defects in clathrincoated vesicle formation and internalization [26]. The mechanism of Myosin VI transport in endocytosis has yet to be fully resolved as both monomers and dimers of Myosin VI have been observed to move processively upon cargo binding $[9,11,27]$.

Although Myosin VI has received intense attention as a result of its unique directionality and its ability to perform distinct functions as a cargo transporter and anchor in the cell, relatively little is known regarding the role of Myosin VI in the nervous system [28]. Myosin VI mutant mice exhibit a decrease in the number of synapses, a decrease in dendritic spine length and an increase in the number of astrocytes as well as impaired synaptic transmission $[28,29]$. In Drosophila, Myosin VI has been shown to contribute to the asymmetric division of neural progenitor cells during embryonic nervous system development [30].

Additionally, a genetic study from our lab identified a potential role for Myosin VI as a modifier of NMJ development [31]. We were therefore interested in understanding the role of Myosin VI at this model synapse and asked how loss of jar affects synaptic function and development. We first observed a significant locomotor defect in severe jar loss of function mutants, and then we used microscopy and electrophysiology to further characterize the role of Myosin VI at the NMJ. We show novel roles for Myosin VI in maintaining proper synaptic vesicle localization to the bouton periphery and in regulating synaptic transmission.

\section{Results \\ Myosin VI protein expression levels in jar loss of function mutants}

The jar alleles used here have been previously characterized [11,32]; we quantified the Myosin VI protein levels by western blot of third instar larval brains and body walls, excluding the mouth parts. The resulting blot revealed reduced Myosin VI levels in all jar loss of function mutants tested (Figure 1), with virtually no Myosin VI found in the most severe $j a r^{322} / D f(3 R)$ crb87-5 zygotic and maternal null allelic combination. When the band density was measured for Myosin VI staining and normalized to the intensity of the loading control, $\beta$ tubulin, a reduction in Myosin VI levels was also observed in two different heterozygote combinations, $j a r^{322} /+$ and $D f(3 R) c r b 87-5 /+$, as compared to the control in blots of both brain and body wall samples, although this is less obvious on the blot. These data are representative of three separate trials of this experiment and serve to confirm the Myosin VI alleles used for this study were loss of function, consistent with previous descriptions of these alleles [32].

\section{Myosin VI mutants exhibit general locomotor defects}

Myosin VI mutant larvae were observed to be sluggish compared to their wild-type counterparts. To characterize any locomotor defects present in jar mutant larvae, larval path length testing was performed on the most severe jar mutant, jar ${ }^{322} / D f(3 R) c r b 87-5 M N$, and a control strain. Larval path length measured over 5 minutes was significantly shorter on both a nutritive and nonnutritive substance for $j a r^{322} / D f(3 R)$ crb87-5 MN larvae $(1.36 \pm 0.19 \mathrm{~cm}, \mathrm{n}=40 ; 5.43 \pm 0.75 \mathrm{~cm}, \mathrm{n}=20)$ compared to the control $(5.16 \pm 0.55 \mathrm{~cm}, \mathrm{n}=40 ; 16.61 \pm$ $1.30, \mathrm{n}=20$ ) (Two-Way ANOVA, $\mathrm{p}<0.0001$, Figure 2). Since the path lengths were shorter on both nutritive and non-nutritive substrates, these results indicate that the jar mutant exhibits general locomotor dysfunction as opposed to a foraging defect, wherein path length would be expected to differ from the control only on a nutritive substrate [33].

\section{Myosin VI contributes to proper synaptic morphology} Although Myosin VI is known to be important for Drosophila embryonic nervous system development [11,30,34], a function for Myosin VI at the NMJ has yet to be described and the locomotor defects observed in the jar maternal nulls mutant may reflect problems at this synapse. NMJ morphology was visualized in jar loss of function mutants by staining neuronal tissues with an FITC-conjugated anti-HRP antibody. Third instar larval NMJs on ventral longitudinal muscles 6 and 7 exhibited a significant decrease in NMJ length for all of the jar loss of function mutants studied compared to the control (Dunn's Multiple Comparison Test, $\mathrm{p}<0.05$, Figure 3 ). The shortest mean NMJ length was observed for $j a r^{322} / D f(3 R)$ crb87-5 larvae, $171.56 \pm 2.29822 \mu \mathrm{m}$ (mean $\pm \mathrm{SE}, \mathrm{n}=24$ NMJs), compared to the control, $293.87 \pm 12.9877 \mu \mathrm{m}(\mathrm{n}=66 \mathrm{NMJs})$. In addition, a significant decrease in Ib bouton number was observed for jar loss of function mutants at muscle 6/ 7 NMJs (Dunnett's Multiple Comparison Test, p < 0.05) (Figure 4). On control NMJs from muscle 6/7, $32.78 \pm$ 1.3025 boutons (mean \pm SE, $n=58$ NMJs) were observed; whereas these synapses in $j a r^{322} / D f(3 R)$ crb87-5 larvae had 


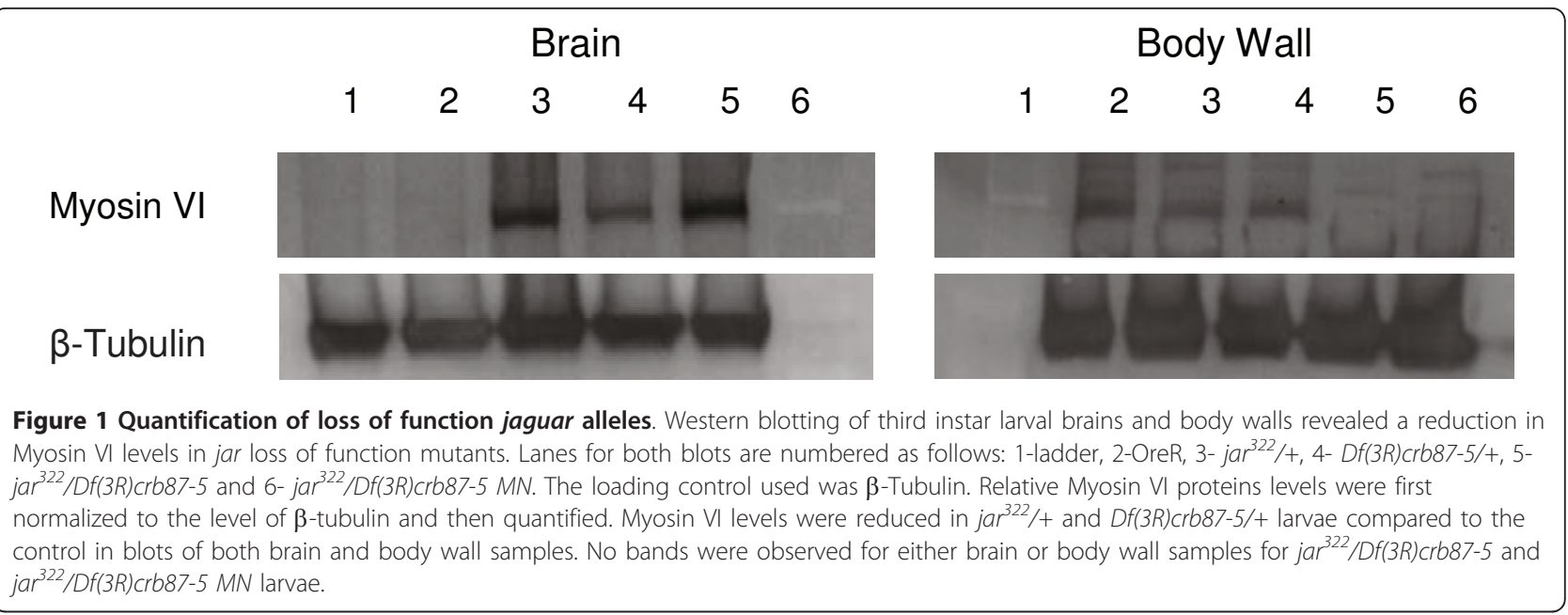

a mean number of $21.02 \pm 0.60573$ boutons $(n=58$ NMJs).

To further investigate the role of Myosin VI in regulating synaptic morphology, staining of the integral vesicle membrane protein Synaptotagmin I was used to visualize synaptic vesicle localization in the boutons of control and jar loss of function mutant larvae. Ultrastructural studies have shown that vesicles are localized to the periphery of boutons, with the centre either void or partially occupied by mitochondria [35]; this gives rise to a characteristic torus (doughnut)-shaped synaptotagmin pattern, with vesicles concentrated in an outer ring of the bouton when viewed with the light microscope [36]. Surprisingly, visualization of synaptotagmin staining in jar mutant boutons revealed a disruption in proper vesicle localization. The majority of $j a r^{322} / D f(3 R) c r b 87-5$ Ib boutons exhibited diffuse synaptotagmin localization across the entire bouton area, rather than the characteristic toruspattern. The differences in synaptotagmin distribution are illustrated by representative control and mutant

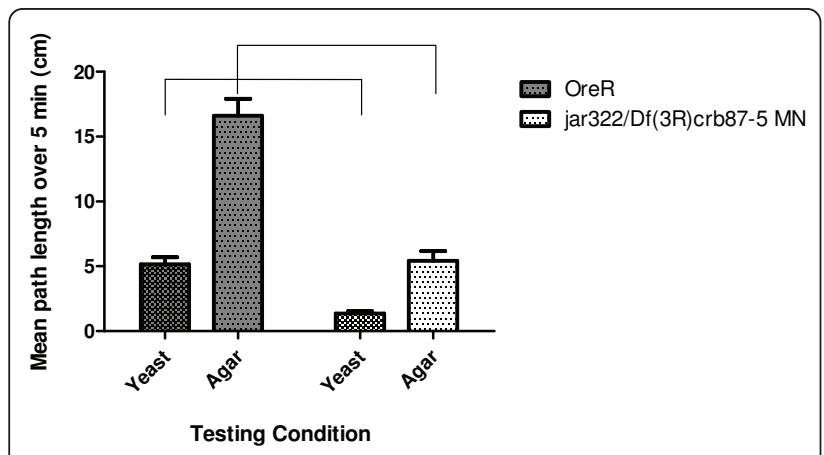

Figure 2 jaguar mutants exhibit general larval locomotor defects. Third instar jar 322 /Df(3R)crb87-5 MN larvae exhibited a significant decrease in path length on both a nutritive and nonnutritive substrate compared to the control (ANOVA, ${ }^{* * *}=p<$ 0.0001). $j a r^{322} / D f(3 R) \operatorname{crb87-5}$ boutons, shown with their corresponding plot profiles (Figure 5A and $5 \mathrm{~B}$ ).

To quantify these observed differences in synaptotagmin localization we calculated the relative difference in fluorescence intensity across a bouton's plot profile [(max intensity-min intensity)/max intensity]. Relative difference in fluorescence intensity was significantly lower across $j a r^{322} / D f(3 R)$ crb87-5 mutant boutons compared to control boutons (Unpaired T-test, $\mathrm{p}<0.001$ ) (Figure $5 \mathrm{C}$ ). However, there was no significant difference in mean fluorescence intensity over total bouton area for both genotypes (Unpaired T-test, $\mathrm{p}>0.05$ ) (Figure 5D). Interestingly, diffuse $j a r^{322} / D f(3 R) c r b 87-5$ boutons were found to be significantly larger than control boutons and $j a r^{322} / D f(3 R) c r b 87-5$ boutons with a normal, torus-shaped distribution of synaptotagmin (Dunn's Multiple Comparison Test, $\mathrm{p}<0.001$ ) (Figure 5E).

To quantify the population of boutons displaying abnormal synaptotagmin distribution, Ib boutons were scored as normal or diffuse for control and jar mutant muscle 6/7 NMJs in third instar larvae. This protocol revealed an increase in the percentage of diffuse Ib boutons for jar mutant NMJs compared to control NMJs (Figure 6). A Chi Square goodness of fit test revealed that all jar mutants significantly differed from the control in number of normal and diffuse Ib boutons ( $\mathrm{p}<$ 0.05). Taken together these data indicate that Myosin VI contributes to proper synaptic development. Specifically, the unexpected diffuse synaptotagmin staining over the Ib bouton centre observed in jar mutants suggests that Myosin VI plays a role in maintaining normal peripheral vesicle localization.

\section{Myosin VI plays a role in basal synaptic transmission}

As the localization of synaptic vesicles is known to be important in basal synaptic function [37], the vesicle mislocalization phenotype observed at jar mutant 

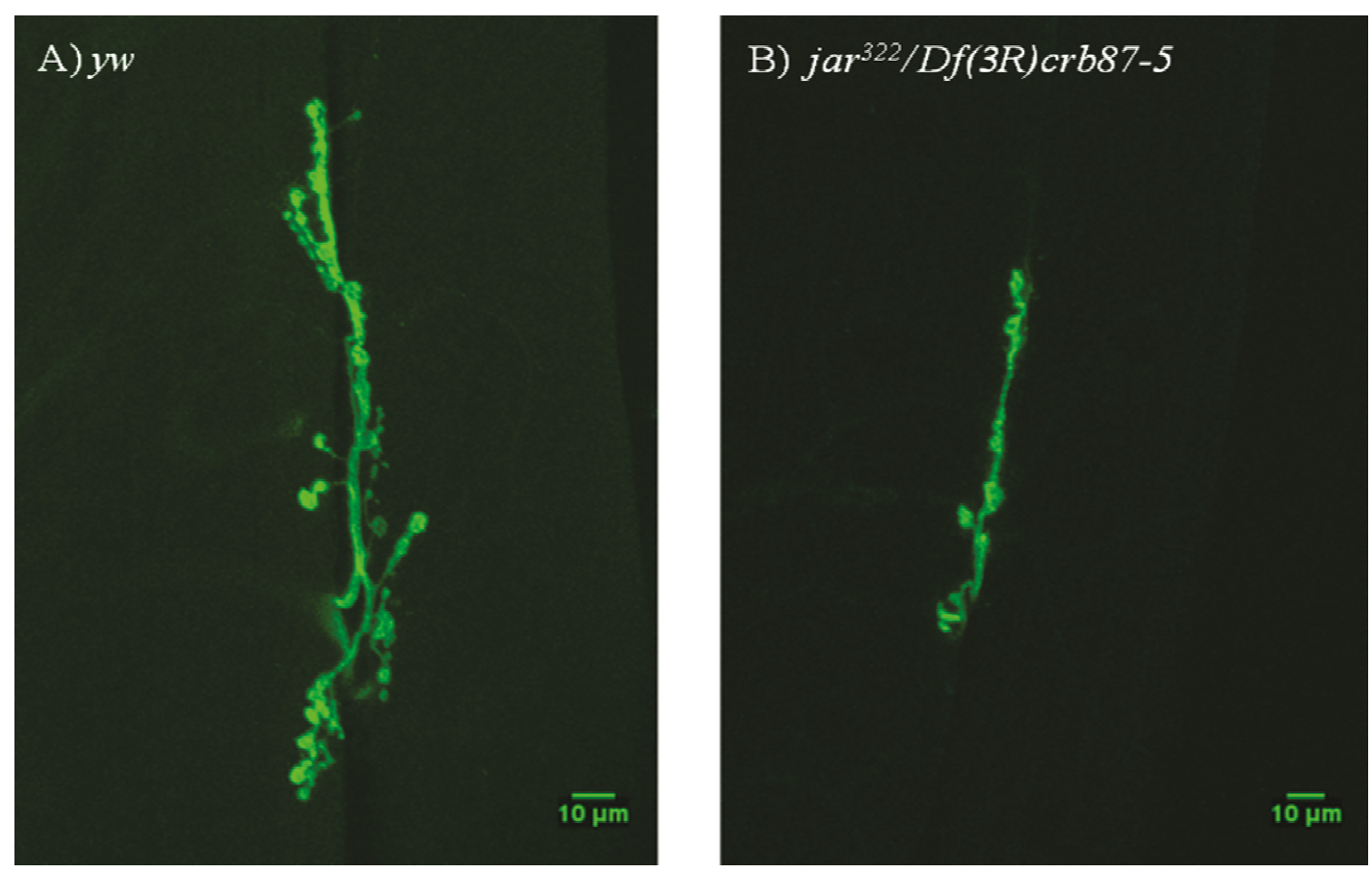

C)

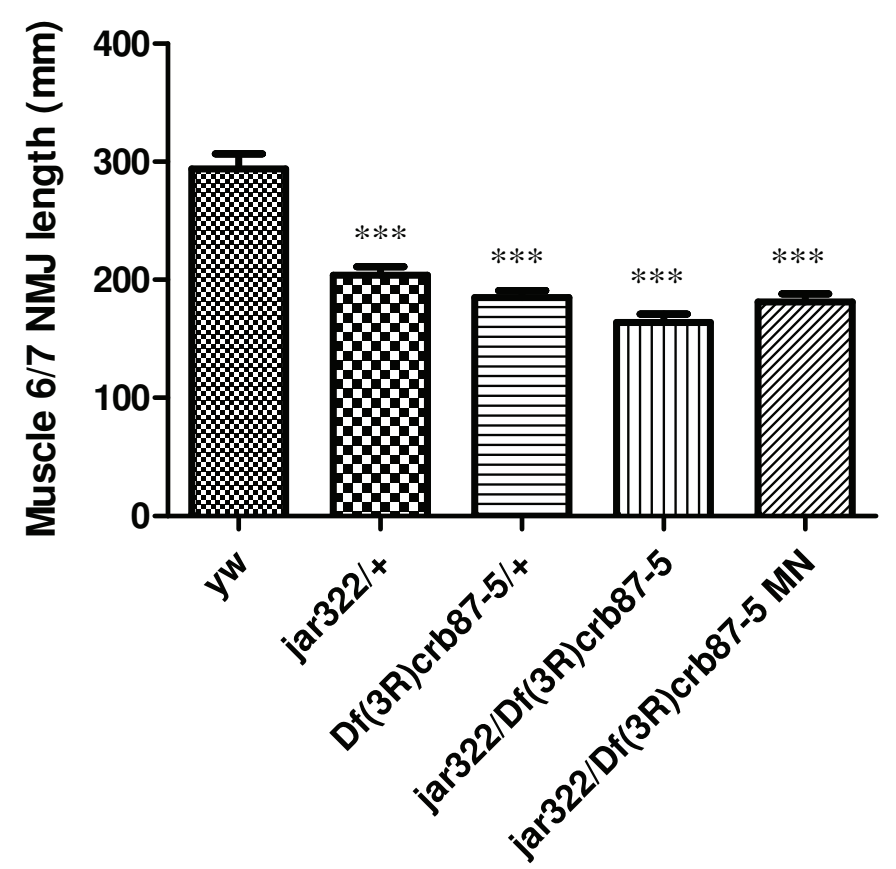

\section{Genotype}

Figure 3 jaguar loss of function mutants display reduced muscle 6/7 NMJ length. Representative NMJs on ventral longitudinal muscles 6 and 7 from third instar Drosophila larvae of the control (A) and the jar loss of function mutant, jar $322 / D f(3 R) c r b 87-5$ (B). All images were acquired at the same magnification using a LSM510 confocal laser microscope. Muscle 6/7 NMJ lengths for the jar loss of function genotypes studied were significantly shorter than the control (Dunn's Multiple Comparison Test, ${ }^{* * *}=p<0.001$ ) (C). Bars represent mean \pm SEM. 


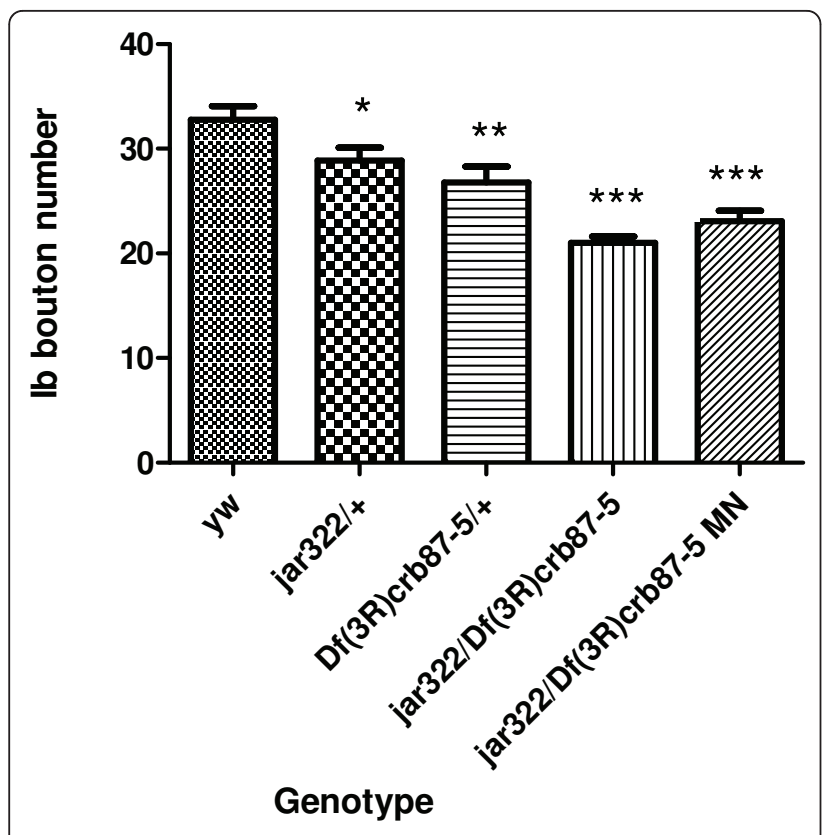

Figure 4 jaguar loss of function mutants exhibited a reduction in bouton number. A significant decrease in Ib bouton number was observed for all jar loss of function mutants studied at muscle 6/7 NMJs in segments $\mathrm{A} 3, \mathrm{~A} 4$ and $\mathrm{A} 5$ compared to the control (Dunnett's Multiple Comparison Test, ${ }^{*}=p<0.05,{ }^{* *}=p<0.01$, $\left.{ }^{* * *}=p<0.001\right)(D)$. Bars represent mean \pm SEM.

synapses may be associated with impaired synaptic transmission. To characterize synaptic physiology at jar mutant synapses, synaptic transmission was assessed by measuring nerve-evoked and spontaneous transmitter release at larval NMJs. Low frequency stimulation allows us to assess the state of the readily releasable pool of vesicles, whereas higher frequency stimulation allows us to probe the recruitment of vesicles from the reserve pool. The ability of the neuron to maintain transmission under sustained high frequency stimulation also allows us to measure the endocytotic ability of the synapse.

Low frequency nerve-evoked responses were collected at $1 \mathrm{~Hz}$ stimulation and the mean EJP amplitude was calculated from 16 stimuli per NMJ. jar mutants lacking a maternal contribution of Myosin VI, jar ${ }^{322} / D f(3 R)$ crb87-5 $M N$, exhibited a significantly lower mean EJP amplitude $(21.34 \pm 2.4572 \mathrm{mV}, \mathrm{n}=16)$ than the control (35.47 $\pm 2.2311 \mathrm{mV}, \mathrm{n}=12$; Dunnett's Multiple Comparison Test, $\mathrm{p}<0.001$ ) (Figure 7A).

Recordings of spontaneous vesicle release, collected for 1 to 2 minutes, were used to calculate mEJP amplitude and frequency. A reduction in mEJP frequency was observed in the more severe jar loss of function mutants, $j a r^{322} / D f(3 R)$ crb87-5 and jar ${ }^{322} / D f(3 R)$ crb87-5 $M N(2.12 \pm 0.238 \mathrm{~Hz}, \mathrm{n}=17$ and $1.79 \pm 0.2104 \mathrm{~Hz}, \mathrm{n}=$ 16 respectively), compared to the control (3.73 \pm 0.2092 $\mathrm{Hz}, \mathrm{n}=14$; Dunnett's Multiple Comparison Test, $\mathrm{p}<$
0.001) (Figure 7C). Average mEJP amplitude was found to be approximately $1 \mathrm{mV}$ for all genotypes studied, with no significant differences between them (ANOVA, $\mathrm{p}>0.05$ ) (Figure 7D).

\section{Myosin VI affects short-term synaptic plasticity}

To further characterize synaptic function at jar mutant synapses, a high frequency stimulation protocol was used to examine changes in synaptic plasticity. The high frequency stimulation protocol was 16 EJPs at $1 \mathrm{~Hz}$, followed by $10 \mathrm{~Hz}$ stimulation for 10 minutes, concluding with $0.1 \mathrm{~Hz}$ stimulation for 10 minutes. In $1 \mathrm{mM}$ extracellular $\mathrm{Ca}^{2+} \mathrm{HL} 3$-saline, the typical pattern of synaptic response to $10 \mathrm{~Hz}$ stimulation began with rapid depression of EJP amplitude, likely due to the depletion of the readily releasable pool (RRP) [38,39]. This was followed by an increase in EJP amplitude, corresponding to mobilization of the reserve pool (RP) due to the presence of residual $\mathrm{Ca}^{2+}$ in the neuronal cytoplasm $[6,38]$, and a subsequent steady decline with continuing high frequency stimulation. For analysis, EJP amplitudes were normalized to the average of the first 16 EJPs collected at $1 \mathrm{~Hz}$ for each recording. No significant differences in depression at the onset of high frequency stimulation were observed between the genotypes (ANOVA, p > 0.05) (Figure 8). The enhancement in EJP amplitude following the initial depression was significantly greater for jar ${ }^{322} / D f(3 R) c r b 87-5$ larvae than the control larvae $(124.14 \pm 5.97126 \%, \mathrm{n}=11$ and $107.49 \pm 2.1921 \%, \mathrm{n}=$ 10 respectively; Dunnett's Multiple Comparison Test, $\mathrm{p}<0.001$ ) (Figure 8). There were no significant differences in maximum EJP amplitude for the jar loss of function heterozygotes relative to the control.

Following high frequency stimulation, post-tetanic potentiation (PTP) is observed as an enhancement of EJP for a short period of time. PTP is presynaptic in origin, caused by an increase in neurotransmitter quanta release, which is attributed to calcium release from intracellular stores that was accumulated during high frequency stimulation $[39,40]$. No significant difference was found in the extent of PTP at $0.1 \mathrm{~Hz}$ for the genotypes studied (ANOVA, p > 0.5) (Figure 8). In addition, there was no significant difference in the amplitude of the last EJP measured at $0.1 \mathrm{~Hz}$ stimulation between genotypes (ANOVA, p > 0.5) (Figure 8).

To further challenge the synapse, the same high frequency stimulation protocol was carried out in $10 \mathrm{mM}$ (supraphysiological) $\mathrm{Ca}^{2+}$ saline. This produced a rapid depression in EJP amplitude at the onset of $10 \mathrm{~Hz}$ simulation, likely due to vesicle depletion in response to high calcium concentrations [39]. The initial depression was followed by a slight recovery and then a continuing decline in EJP amplitude for the remainder of the $10 \mathrm{~Hz}$ stimulation. The initial depression in EJP amplitude measured 

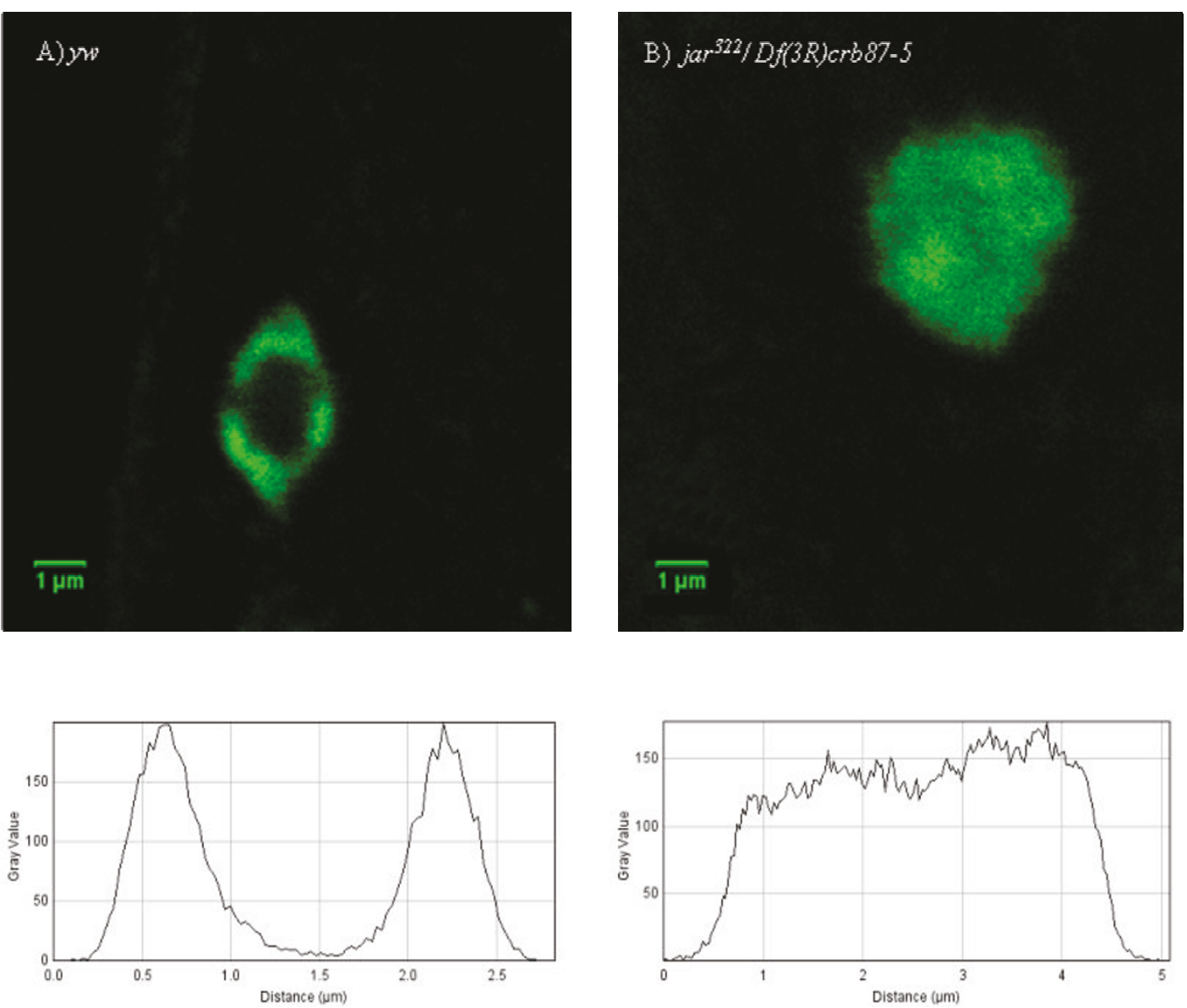

C)

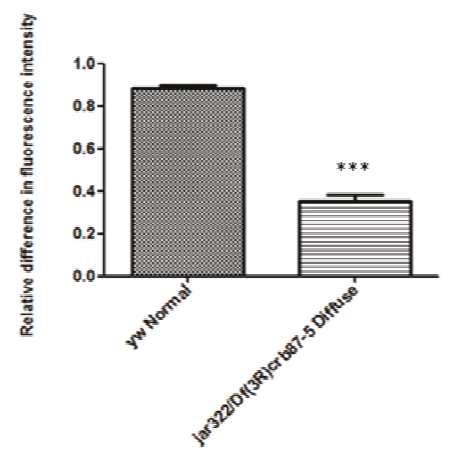

Genotype and Ib Bouton Phenotype
D)

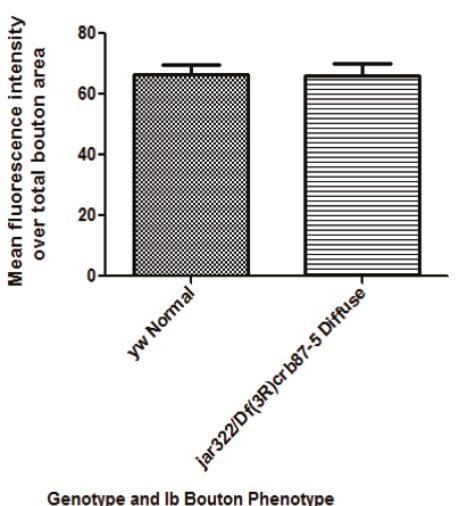

E)

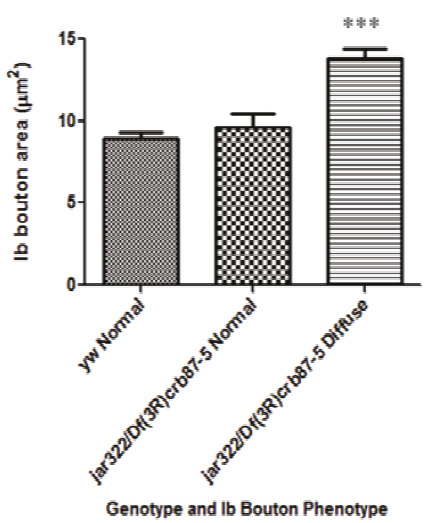

Figure 5 Synaptic vesicles are mislocalized at jaguar mutant boutons as revealed by immunostaining against synaptotagmin. A representative control $\mathrm{lb}$ bouton and mutant $j a r^{322} / \mathrm{Df}(3 \mathrm{R}) \mathrm{crb} 87-5 \mathrm{lb}$ bouton are shown with their corresponding plot profiles (A and B). There was a significant reduction in relative difference in fluorescence intensity across jar ${ }^{322} / \mathrm{Df}(3 R)$ crb87-5 mutant boutons $(n=13)$ compared to the control boutons ( $n=25$; Unpaired T-test, $\left.{ }^{* * *}=p<0.001\right)$ (C). Mean fluorescence intensity did not differ significantly between the mutant ( $n=$ $48)$ and the control $(n=64$; Unpaired T-test, $p>0.05)$ (D). Diffuse jar ${ }^{322} / \mathrm{Df}(3 R)$ crb87-5 boutons $(n=53)$ were significantly larger than control boutons $(n=64)$ and jar ${ }^{322} / \mathrm{Df}(3 R)$ crb87-5 boutons with a normal, doughnut-shaped distribution of synaptotagmin $(n=27$, Dunn's Multiple Comparison Test, ${ }^{* * *}=p<0.001$ ) (E). Boutons used for this study were from muscle 6/7 NMJs in segments A3, A4 and A5. Bars represent mean \pm SEM. 

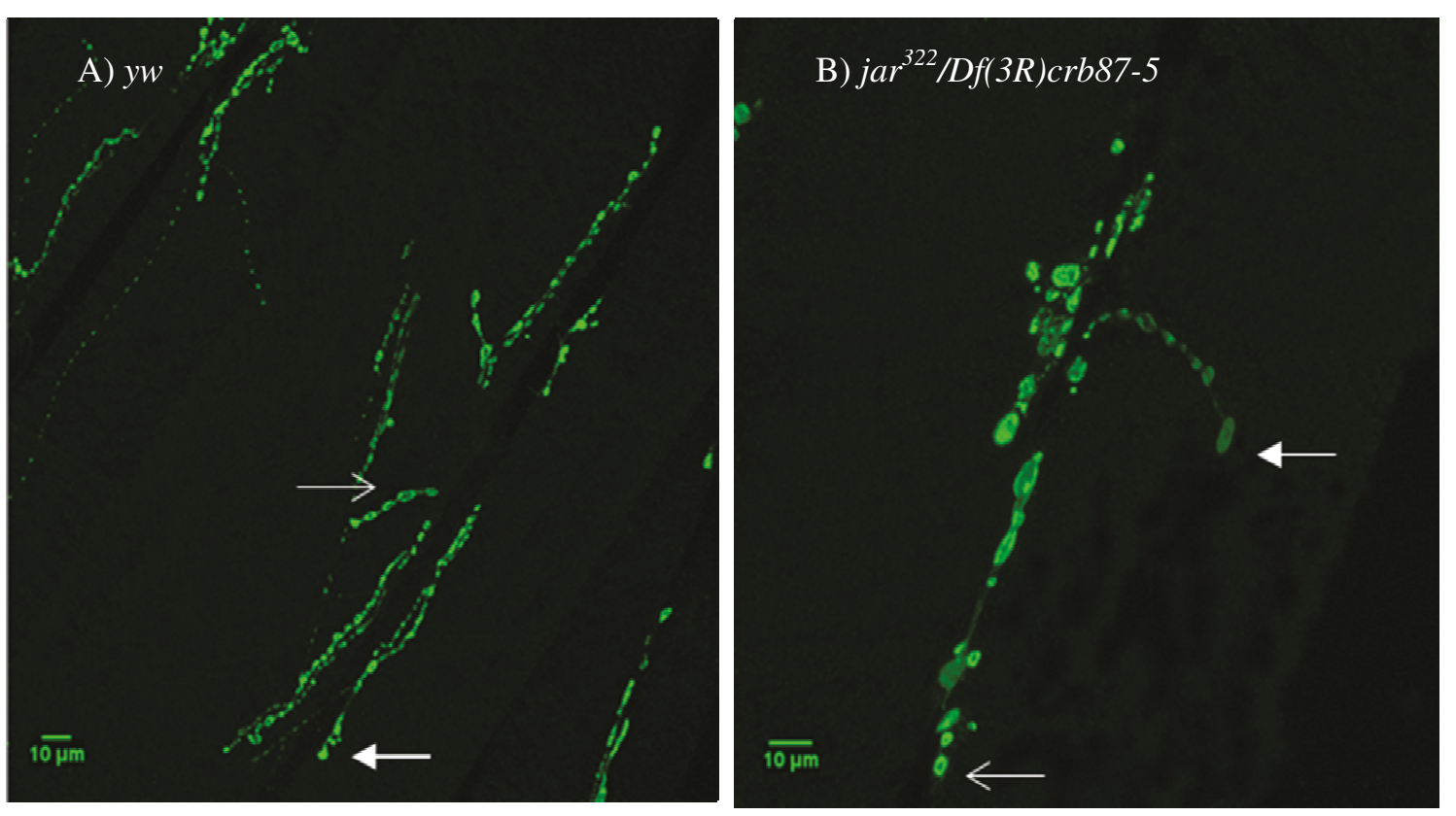

C)

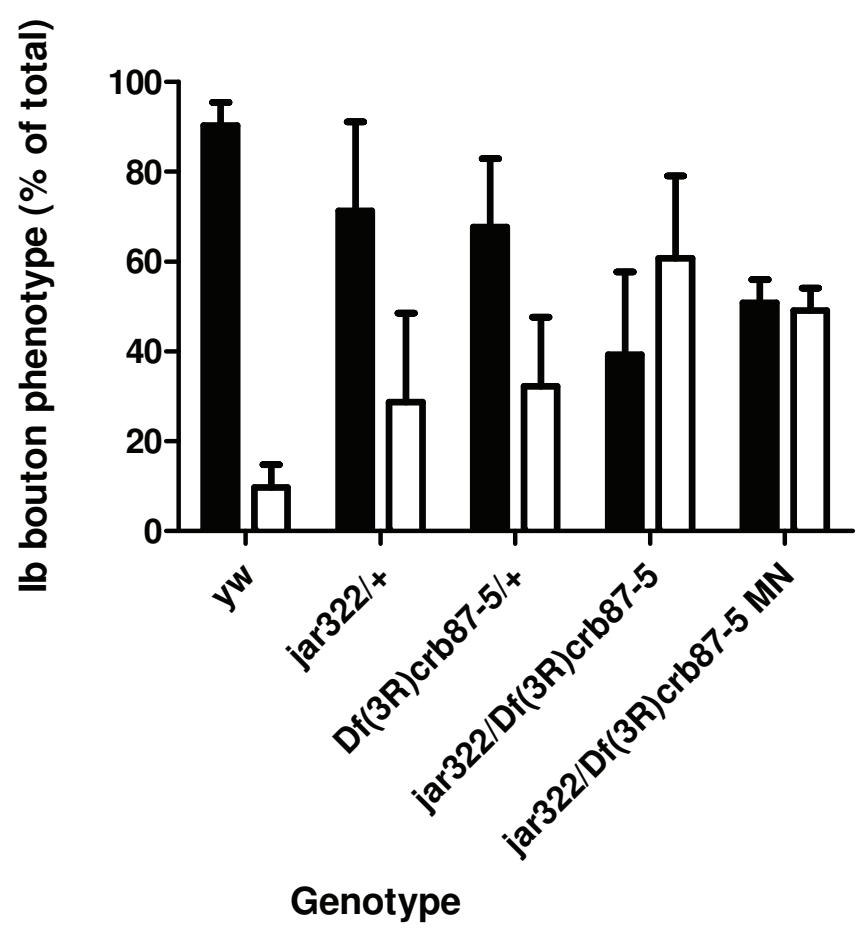

Figure 6 Boutons exhibiting vesicle mislocalization increased corresponding to the severity of jaguar loss of function. Immunostaining against synaptotagmin of NMJs on muscles 6/7 of control and jar $322 / D f(3 R)$ crb87-5 third instar larvae revealed different proportions of boutons exhibiting normal and diffuse synaptotagmin staining (A and B). Images were taken at different magnifications to visualize the entire NMJ. Open arrows indicate the normal, doughnut-shaped pattern of synaptotagmin localization and closed arrows indicate the diffuse pattern of synaptotagmin localization. Scoring the synaptotagmin distribution phenotype revealed a reduction in the percentage of normal boutons and an increase in the percentage of diffuse boutons in jar loss of function mutants compared to the control (C). Number of boutons analyzed were $y w$ $\mathrm{n}=48, j a r 322 /+\mathrm{n}=38, \mathrm{Df}(3 \mathrm{R}) \mathrm{crb87}-5 /+\mathrm{n}=28, j a \mathrm{r}^{322} / \mathrm{Df}(3 \mathrm{R}) \mathrm{crb87}-5 \mathrm{n}=58$ and jar ${ }^{322} / \mathrm{Df}(3 R) \mathrm{crb} 87-5 \mathrm{MN} \mathrm{n}=18$. Boutons used for this study were from muscle 6/7 NMJs in segments A3, A4 and A5. Bars represent mean \pm SEM. 


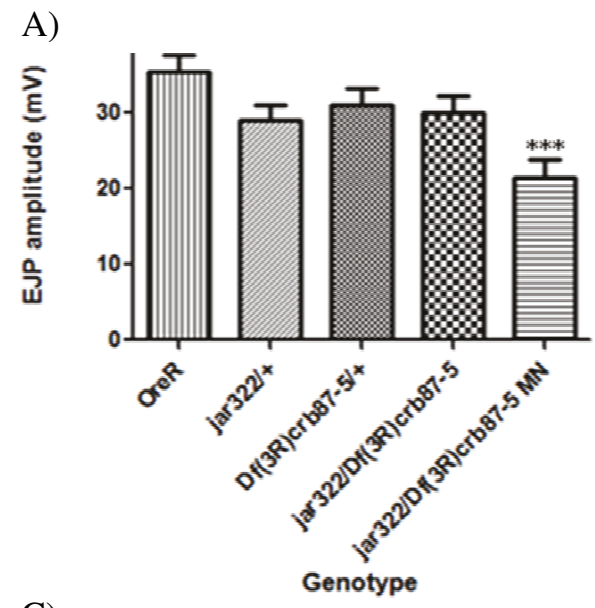

C)

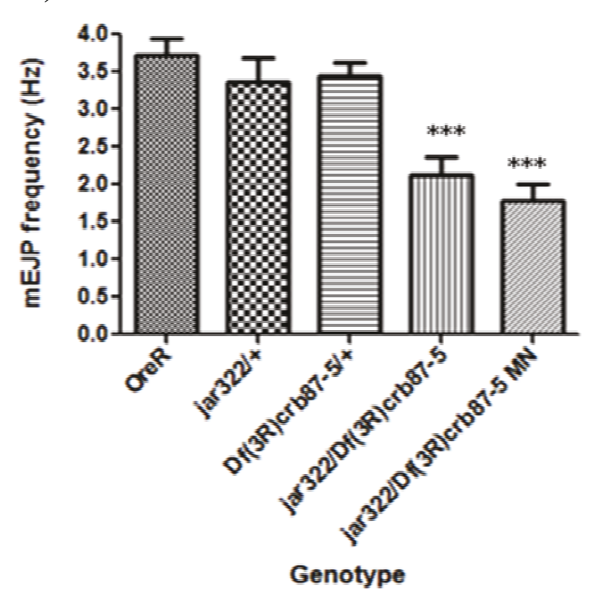

D)

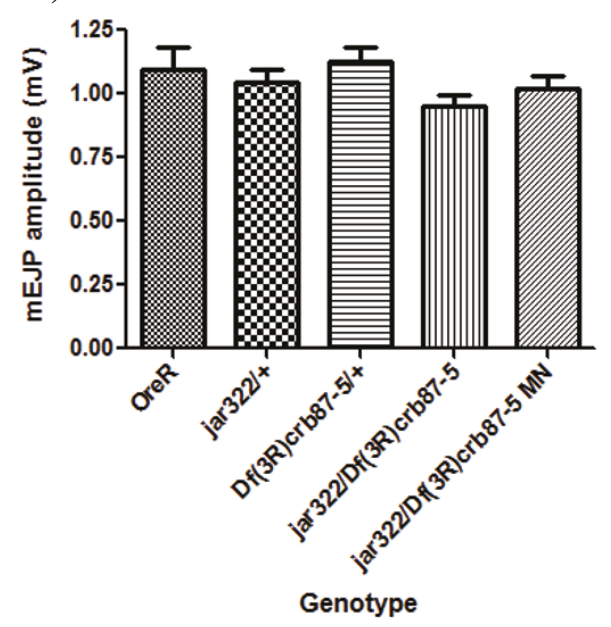

B)
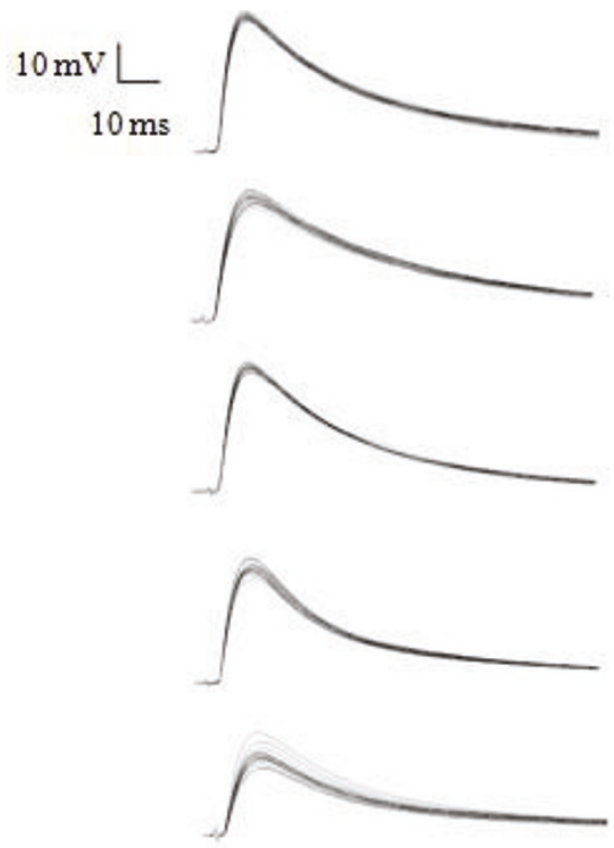

E)

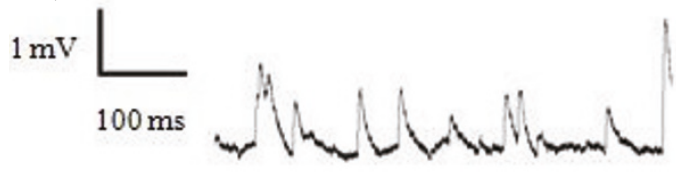

Figure 7 jaguar loss of function mutants exhibited defects in basal synaptic transmission. Electrophysiological recordings from muscles 6/ 7 of third instar larvae revealed EJP amplitude was significantly reduced in the most severe jar mutant, jar ${ }^{322} / D f(3 R) c r b 87-5$ MN, compared to the control (Dunnett's Multiple Comparison Test, $\left.{ }^{* * *}=p<0.001\right)$ (A). Sample traces of EJP recordings taken at $1 \mathrm{~Hz}$, shown in the same order as listed for the graphs (B). mEJP frequency was significantly lower for jar ${ }^{322} / \mathrm{Df}(3 \mathrm{R}) \mathrm{crb87-5}$ and jar $\mathrm{r}^{322} / \mathrm{Df}(3 \mathrm{R}) \mathrm{crb87-5} \mathrm{MN}$ larvae compared to the control larvae (Dunnett's Multiple Comparison Test, ${ }^{* * *}=\mathrm{p}<0.001$ ) (C). There was no significant difference in mEJP amplitude between jar mutants and the control (ANOVA, p > 0.05) (D). Sample traces of electrophysiological recordings from muscles $6 / 7$ over 2 seconds, shown in the same order as listed for the graphs (E). Bars represent mean \pm SEM. 


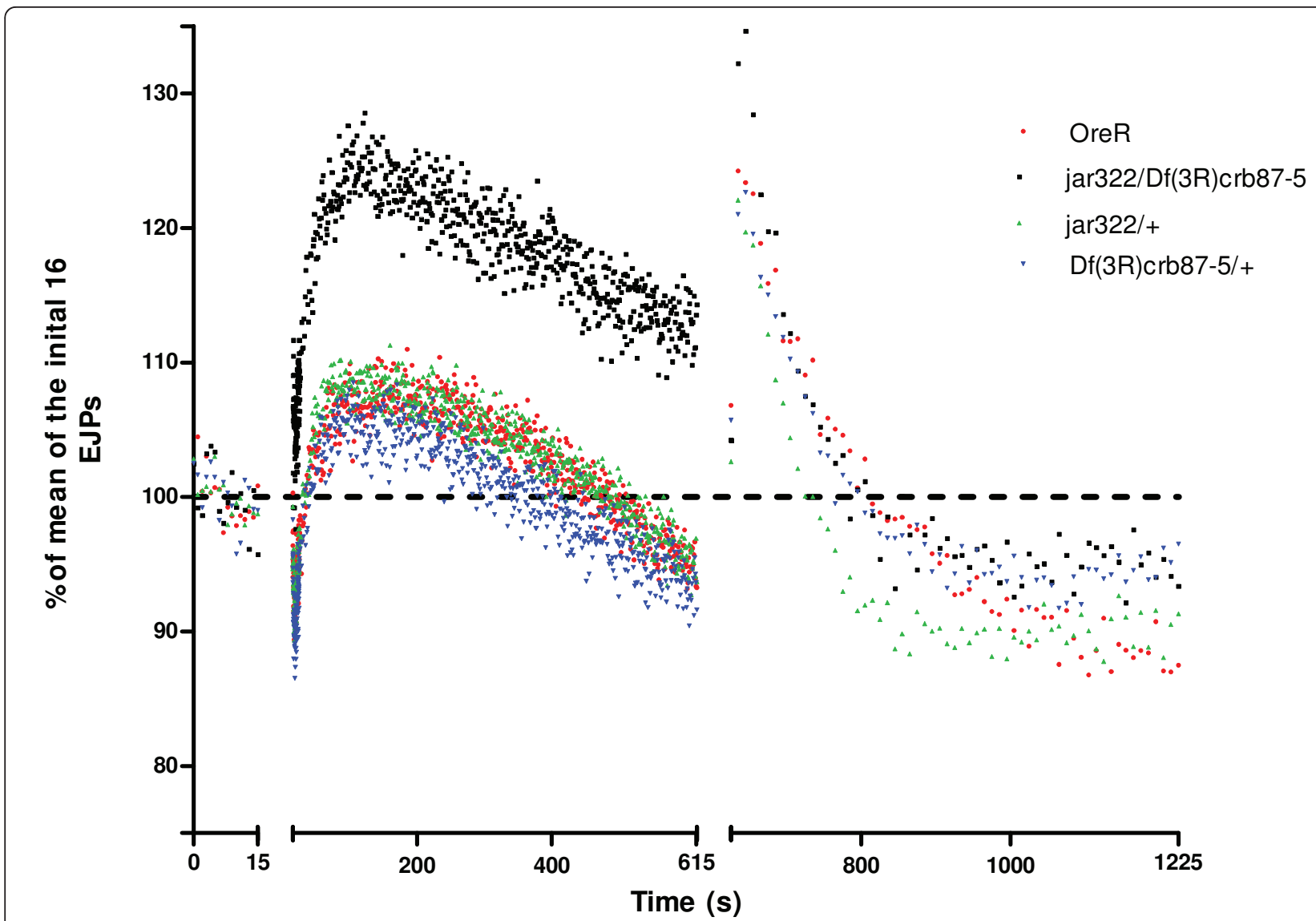

Figure 8 High frequency stimulation in $1 \mathrm{mM} \mathrm{Ca}^{2+}$ saline revealed enhanced potentiation in $j a r^{322} / D f(3 R) c r b 87-5$ mutants. Data is shown as a percent of the average of the first 16 evoked junctional potentials (EJPs) recorded at $1 \mathrm{~Hz}$. This baseline recording of 16 EJPs at $1 \mathrm{~Hz}$ was followed by a stimulation of $10 \mathrm{~Hz}$ recorded over 10 minutes and terminated with a 10 minute recording at $0.1 \mathrm{~Hz}$. Following the first 25 seconds from the onset of $10 \mathrm{~Hz}$ stimulation, every tenth recording is shown. A significant enhancement in EJP amplitude was observed for $j^{322} / \mathrm{Df}(3 R)$ crb87-5 larvae during the first three minutes of the high frequency protocol compared to the control (Dunnett's Multiple Comparison Test, $\mathrm{p}<0.001)$.

relative to EJP amplitude at the onset of high frequency stimulation was significantly greater for $\mathrm{jar}^{322} / D f(3 R)$ crb87-5 larvae than the control larvae $(0.63 \pm 0.04, \mathrm{n}=12$ and $0.45 \pm 0.02, \mathrm{n}=8$ respectively; ANOVA, $\mathrm{p}<0.01$ ) (Figure 9). There were no significant differences in initial depression among the other genotypes tested (ANOVA, $\mathrm{p}$ $>0.05$ ). Recovery of EJP amplitude at $10 \mathrm{~Hz}$ stimulation was quantified as a percent increase from the lowest EJP amplitude during the initial depression to the largest EJP amplitude observed during the recovery period. There was no difference in recovery among control and jar loss of function larvae following $10 \mathrm{~Hz}$ stimulation (ANOVA, p > 0.05). Additionally, no difference in recovery of EJP amplitude was found among genotypes at $0.1 \mathrm{~Hz}$ stimulation (ANOVA, $\mathrm{p}>0.05$ )

\section{Discussion}

This work has demonstrated a novel role for Myosin VI in proper synaptic development, vesicle localization, and synaptic transmission at the Drosophila NMJ. Severe jar loss of function mutants exhibited significant defects in larval locomotor behavior prompting our investigations of Myosin VI at the NMJ. Imaging of synaptic morphology revealed that Myosin VI is important for NMJ development: a reduction in NMJ length and a decrease in bouton number were observed at jar loss of function mutant nerve terminals.

Although Myosin VI function in the vesicle cycle has been implicated in mammalian cells, this report provides the first evidence that Myosin VI is important for maintaining normal peripheral vesicle localization at the bouton [25]. In Drosophila, there are four types of boutons, which are the sites of neurotransmitter release at the NMJ, and they differ in their morphological and chemical properties [41]. Of interest for this study were the largest synaptic boutons found at type I axon terminals, which are present at all NMJs of mature larvae [42]. Visualization of synaptotagmin staining using confocal imaging 


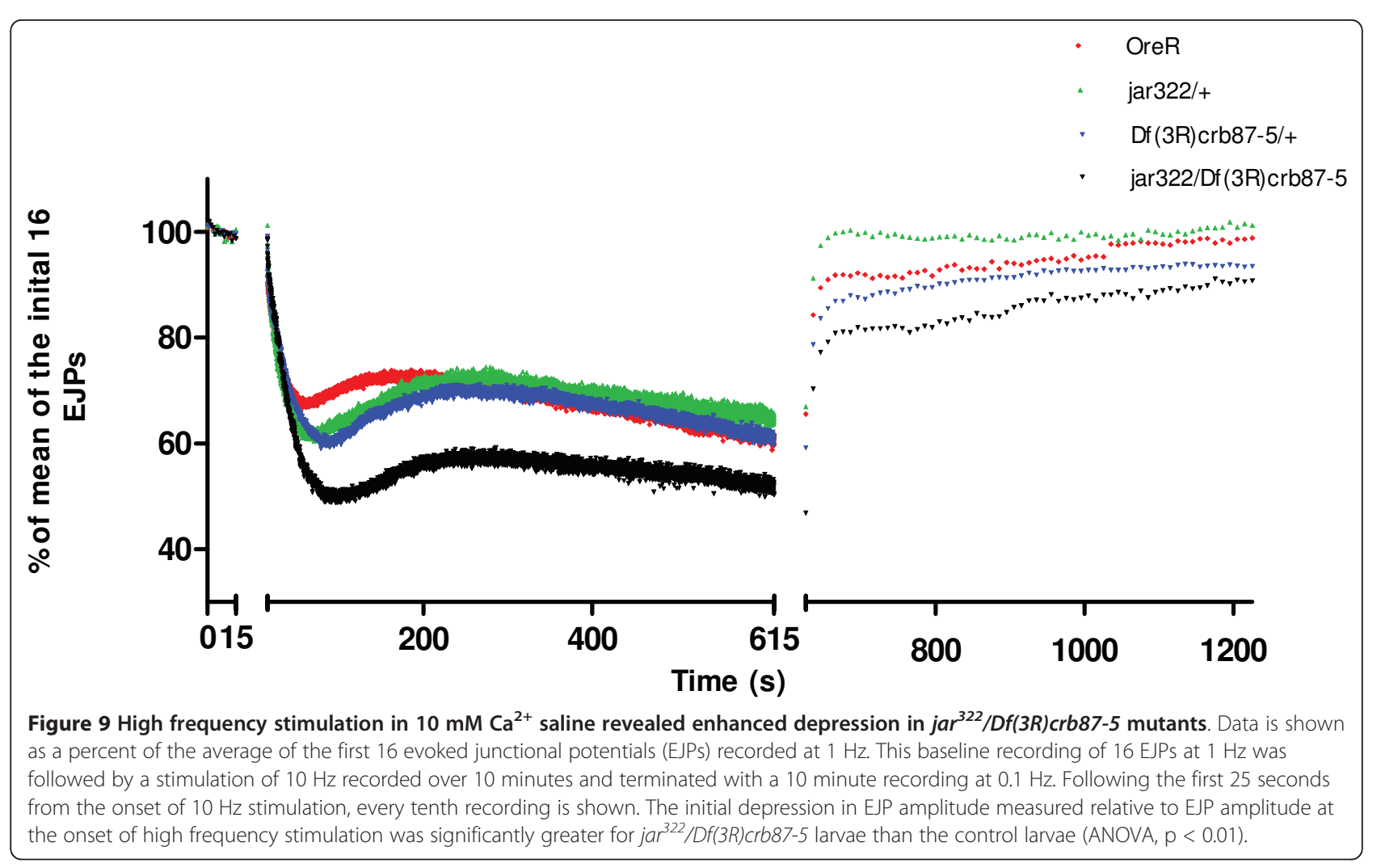

revealed a mislocalization of synaptic vesicles in jar mutant boutons. An increasing number of jar mutant boutons, corresponding to the severity of Myosin VI loss of function, were found to exhibit diffuse staining over the entire bouton area as opposed to the doughnutshaped staining pattern present in control boutons. Bouton centre occupancy has previously been observed at Drosophila NMJs of larvae lacking synapsin, a phosphoprotein that reversibly associates with vesicles, using FM1-43 loading under low frequencies [43]. EM analysis confirmed that in synapsin knockouts there was a spread of vesicles into the bouton centre, accompanied by a reduction in the size of the reserve pool [44]. Thus, synapsin is thought to function in maintaining the peripheral distribution of vesicles in Ib boutons [44]. Likewise, the unexpected diffuse synaptotagmin staining of jar mutant boutons suggests Myosin VI participates in restricting vesicles to the bouton periphery. It is possible that Myosin VI is functioning as a regulator of the actin cytoskeleton at the synapse. Mutant studies have revealed that the presynaptic actin cytoskeleton is required for proper synaptic morphogenesis [45]. Myosin VI has already been shown to function in regulating the actin cytoskeleton during the process of spermatid individualization, by acting either as a structural cross-linker or as an anchor at the front edge of the actin cone, and during nuclear divisions in the syncytial blastoderm $[15,46]$.
However, live imaging of actin dynamics at the synaptic boutons revealed no major defects in the actin cytoskeleton at jar loss of function mutant nerve terminals.

To assess whether the morphological defects in vesicle localization observed at jar mutant synapses impact synaptic transmission, electrophysiological assays with different stimulation paradigms were used to recruit vesicles from different functional pools. Our data add to the knowledge of this protein's physiological role at synapses. Myosin VI mutant mouse hippocampal neurons exhibit defects in the internalization of the $\alpha$-amino-3-hydroxy-5-methyl-4-isoxazole propionic acid-type glutamate receptor, responsible for fast glutamatergic transmission, suggesting Myosin VI normally plays a role in AMPAR endocytosis [28]. In addition, basal synaptic transmission is reduced in Myosin VI deficient mouse hippocampal slices compared to wild-type controls [29]. Electrophysiological experiments also indicate that Myosin VI mediates glutamate release induced by brain-derived neurotrophic factor, which is known to modulate synaptic transmission and plasticity in the mammalian central and peripheral nervous system [29].

Our study is the first to show that Myosin VI's role in synaptic transmission involves mobilization of vesicles from different functional pools, indicating that Myosin VI is important for synaptic plasticity. At the Drosophila NMJ, three pools of vesicles with differential release properties have been identified using FM1-43 staining 
loaded by various stimulation protocols ([47], reviewed by [48]). The immediately releasable pool (IRP), representing approximately $1 \%$ of all vesicles at the NMJ, consists of vesicles docked and primed at active zones for immediate release and experiences rapid depletion within a few stimuli $[48,49]$. The readily releasable pool (RRP), making up 14 to $19 \%$ of all vesicles at the NMJ, is mobilized by moderate stimulation of $\leq 3 \mathrm{~Hz}$ and maintains exo/endocytosis at these stimulation frequencies [49]. The reserve pool (RP) represents the vast majority of vesicles, 80 to $90 \%$, and is mobilized upon depletion of the RRP [49]. Recruitment from the RP occurs with high frequency stimulation of $\geq 10 \mathrm{~Hz}$ [50]. Spontaneous release was reduced in the most severe jar loss of function mutants. Evoked response at $1 \mathrm{~Hz}$ stimulation was also reduced in the jar maternal null mutant. Although less severe jar mutants exhibited a significant decrease in bouton number, they did not experience an accompanying reduction in evoked potential amplitude at low frequency stimulation, suggesting that other homeostatic mechanisms are important for maintaining synaptic strength [51]. The impaired synaptic response in the jar maternal null mutant may be due to a reduction in the probability of RRP vesicle release or in RRP size. If Myosin VI functions to anchor synaptic vesicles, it may act on the RRP to ensure vesicles are localized in manner that makes them readily available for release. Thus, in jar maternal null mutants the reduction in EJP amplitude may occur because a significant number of vesicles were displaced from areas of higher probability release. Alternately, RRP pool size may be reduced at jar mutant synapses.

Different synaptic vesicle pool properties, such as rate of recruitment of the RP in response to high frequency stimuli, may translate to changes in short-term synaptic plasticity [37]. The increase in EJP amplitude observed at $10 \mathrm{~Hz}$ stimulation in $1 \mathrm{mM} \mathrm{Ca}^{2+}$ saline may be attributable to enhanced mobilization of the RP for $j a r^{322} / D f(3 R)$ crb87-5 NMJs [44]. Filamentous actin has been implicated in RP mobilization as cytochalasin D, an inhibitor of actin polymerization, has been shown to reduce RP dynamics [47]. This suggests translocation from the RP to the RRP may be mediated by an actin-based myosin motor protein. If Myosin VI functions as a synaptic vesicle tether to regulate recruitment from the RP pool, RP vesicles would be more readily mobilized and transitioned into the RRP upon high frequency stimulation in jar loss of function mutants. Consistent with the idea that RP vesicles were more rapidly incorporated into the $\mathrm{RRP}$, a greater initial depression is observed at $\mathrm{jar}^{322} / \mathrm{Df}$ (3R)crb87-5 mutant synapses during high frequency stimulation in $10 \mathrm{mM} \mathrm{Ca}^{2+}$ saline corresponding to the depletion of vesicles at high calcium concentrations. Taken together, the data suggest that Myosin VI mediates synaptic transmission and short-term plasticity by regulating the mobilization of synaptic vesicles from different functional pools. In mammalian cells, Myosin VI has been implicated as a mediator of vesicle endocyctosis and has been shown to transport uncoated vesicles through the actin-rich periphery to the early endosome [25]. Our experiments, however, indicate that endocytosis is not likely affected at jar mutant synapses. Typically, endocytotic mutants are unable to maintain synaptic transmission in response to high frequency stimulation $[52,53]$, whereas our Myosin VI loss of function mutants exhibited enhanced EJP amplitude observed at $10 \mathrm{~Hz}$ stimulation in $1 \mathrm{mM} \mathrm{Ca}^{2+}$ saline. Additional experiments are required to confirm that Myosin VI is functioning as a vesicle tether. Fluorescence recovery after photobleaching analysis can be used to examine the effect of Myosin VI on synaptic vesicle mobility. If Myosin VI is functioning as a vesicle tether, synaptic vesicle mobility is expected to be increased in jar mutants compared to controls.

\section{Conclusions}

In summary, the present work shows that Myosin VI is important for proper synaptic morphology and physiology at the Drosophila NMJ. Myosin VI function in peripheral vesicle localization at the bouton may underlie its contribution to basal synaptic transmission and expression of synaptic plasticity. Future work will address the mechanism by which Myosin VI performs its roles at the synapse, whether as a vesicle tether or by some other involvement in vesicle trafficking.

\section{Methods \\ Drosophila stocks}

All fly strains and crosses were maintained on Bloomington standard medium (http://flystocks.bio.indiana. edu/Fly_Work/media-recipes/bloomfood.htm) supplemented with yeast paste at room temperature. The Myosin VI loss of function alleles used in this study were $j a r^{322}$ and $D f(3 R) c r b 87-5$ (maintained as stocks over Tm3, Sb Ser GFP). jar ${ }^{322}$ is a null allele that deletes the entire Myosin VI coding region and some of the neighboring gene, CG5706 [32]. When homozygous, $j^{322}$ is lethal in first or second instar larvae due to the loss of CG5706 function [32]. $D f(3 R)$ crb87-5 is a deletion that removes most of the amino acid coding sequences of the jar gene, through to exon 13 of 17 [32]. jar $322 / D f(3 R)$ crb87-5 animals were produced by crossing together $\mathrm{jar}^{322} / \mathrm{Tm} 3, \mathrm{Sb}$ Ser GFP and $D f(3 R)$ crb87-5/Tm3, Sb Ser GFP flies, and then selecting against the GFP balancer chromosomes. Myosin VI maternal null animals, designated $j a r^{322} / D f(3 R) c r b 87-5$ $M N$, were generated by crossing $j a r^{322} / D f(3 R)$ crb87-5 females to $\mathrm{jar}^{322} / \mathrm{Tm} 3, \mathrm{Sb}$ Ser GFP males, and then 
selecting non-fluorescent larvae. Larvae from $y w$ flies were used as a control for staining experiments, and from Oregon R (OreR) flies for electrophysiological experiments.

\section{Western blots}

Protein extracts were prepared in ice-cold homogenization buffer with proteinase inhibitor. Four body walls or ten brains from third instar larvae per genotype were used. The larval preparations were manually homogenized and pulsed in the centrifuge. This was followed by the addition of $0.5 \mathrm{M}$ Dithiotreitol and $2 \mathrm{x}$ loading buffer. Larval preparations were heated at $100^{\circ} \mathrm{C}$ for 5 minutes prior to loading. Samples were run on a $6 \%$ SDS PAGE gel using modified procedures from [54]. Gels were run for 1.5 hours at $70 \mathrm{~V}$, followed by electropheretic transfer to polyvinylidene difluoride membrane at $350 \mathrm{~mA}$ for 55 minutes. Membranes were incubated with 1:20 mouse anti-myosin VI antibody (gift from Kathy Miller) and 1:1000 anti-tubulin antibody (Hybridoma Bank, University of Iowa). HRP-conjugated goat anti-mouse antibody (BIORAD) was used at a dilution of 1:3000. Resultant bands were visualized using STORM Scanner Control (Molecular Dynamics). The stained bands were now visible for further analysis using Image J. Relative protein levels were quantified and compared.

\section{Test of general larval locomotion}

Larval path length testing was used to assess changes in general larval locomotion in $j a r^{322} / D f(3 R) \operatorname{crb} 87-5 \mathrm{MN}$ larvae and were performed as described by [55]. Briefly, individual third instar larvae ( $96 \pm 2 \mathrm{~h}$ posthatching) were placed onto a layer of yeast paste in a circular well. Each well was covered with a Petri-dish lid and after 5 minutes, the paths traveled by the larvae were traced onto the Petri lids. These path lengths were used to calculate the mean path length in ImageJ for the mutant and control larvae, which were tested concurrently. Similarly, path length was tested on a non-nutritive substrate, $0.4 \%$ agar. Total distance traveled on agar was measured by placing individual third instar larvae $(96 \pm 2 \mathrm{~h}$ posthatching) in the center of agar-coated Petri-dishes, which were then covered with the Petri-dish lids. After 5 minutes, the path traveled by the larvae was traced onto the Petri lids and quantified as described above.

\section{Immunocytochemistry}

For immunostaining, third instar larvae were dissected in HL3 saline, fixed in 4\% EM grade formaldehyde in phosphate buffered saline (PBS) for 15 minutes and then transferred to an Eppendorf tube with 5 to 7 other larvae. For NMJ visualization, larvae were stained with FITC-conjugated goat anti-HRP antibody, (1:1000, MP Biomedical; Solon, $\mathrm{OH}$ ), as described by [56]. For synaptic vesicle labeling, larvae were stained with 1:1000 rabbit polyclonal anti-synaptotagmin antibody, followed by the secondary antibody, AlexaFluor ${ }^{\circledR} 488$ goat antirabbit IgG, (1:1000, Invitrogen), as outlined by [1]. Following staining, larvae were mounted onto slides in Vectashield (Vector Laboratories; Burlington, ON).

Images were obtained using a LSM510 (Carl Zeiss) confocal laser microscope with a $200 \mathrm{~mW}$ Argon laser. To image NMJs, Z-sections were collected for NMJ 6/7, 2 per larvae, at $1 \mu \mathrm{m}$ intervals through the $20 \mathrm{x}$ air lens at 2 times magnification. These images were projected onto a single plane and NMJs were measured (scale: 2.223 pixels $/ \mu \mathrm{m}$ ) using Image J. For synaptotagmin staining, images for individual boutons were obtained under constant imaging parameters, including gain, zoom, pinhole size and laser output, to allow for proper comparative analysis. To analyze synaptotagmin staining in individual boutons, a rectangular cross section of the bouton was taken and a plot profile was generated for this region using Image $\mathrm{J}$.

\section{Electrophysiology}

Wandering third instar larvae were dissected in HL3 saline to which calcium was added for a final concentration of $1 \mathrm{mM}$ [57]. Intracellular electrophysiological recordings for muscle 6 or 7 recordings were taken from abdominal segments A3, A4 and A5, and analyzed as described by [58]. All muscles selected for analysis had initial resting potentials between -60 and $-75 \mathrm{mV}$. Baseline synaptic transmission was characterized by recording miniature excitatory junctional potentials (mEJPs) for 1 to 2 minutes and 16 evoked junctional potentials (EJPs) stimulated at $1 \mathrm{~Hz}$. High frequency stimulation was performed on larvae bathed in either $1 \mathrm{mM} \mathrm{Ca}^{2+} \mathrm{HL} 3$ or 10 $\mathrm{mM} \mathrm{Ca}^{2+}$ modified HL3, the composition of which is as follows (in $\mathrm{mM}$ ): $70 \mathrm{NaCl}, 5 \mathrm{KCl}, 10 \mathrm{MgCl}_{2}, 10 \mathrm{NaHCO}_{3}$, $10 \mathrm{CaCl}_{2}, 115$ sucrose, 5 Trehalose, and 5 HEPES $(\mathrm{pH}$ 7.2). The stimulation protocol used for high frequency recordings was 16 EJPs at $1 \mathrm{~Hz}$, followed by $10 \mathrm{~Hz}$ stimulation for 10 minutes, and concluded with $0.1 \mathrm{~Hz}$ stimulation for 10 minutes. Muscle resistance was recorded for both protocols to ensure it was within normal range, 5 to $10 \mathrm{~m} \Omega$ [59]. In all experiments, a maximum of two recordings were used per larvae and a minimum of 8 larvae were used.

\section{Statistical analysis}

All statistical analyses were performed in GraphPad Prism Software 5. A significance level of $\mathrm{p}<0.05$ was used for all experiments.

\section{Acknowledgements and Funding}

The authors acknowledge the generous gift of Myosin VI antisera from Kathryn Miller, Washington University of St. Louis. We thank Marla 
Sokolowski, University of Toronto Mississauga, for use of her lab to measure larval path length and Bianco Marco for his help with the behavioral techniques. We thank the Bloomington Stock Centre for fly lines. This work was funded by grants (BAS and SC) and scholarships (MK) from the Natural Sciences and Engineering Research Council of Canada and the Canada Research Chairs program (BAS).

\section{Authors' contributions}

The experiments were conceived and designed by MK and BAS; MK performed all experiments, analyzed the data, and assembled figures; DM and SC performed initial observations of jar mutant phenotypes; MK and BAS wrote and edited the manuscript. All authors have read and approved the final manuscript.

\section{Competing interests}

The authors declare that they have no competing interests.

Received: 25 May 2011 Accepted: 11 July 2011 Published: 11 July 2011

\section{References}

1. Nunes P, Haines N, Kuppuswamy V, Fleet DJ, Stewart BA: Synaptic vesicle mobility and presynaptic F-actin are disrupted in a N-ethylmaleimidesensitive factor allele of Drosophila. Mol Biol Cell 2006, 17(11):4709-4719.

2. Seabrooke S, Qiu X, Stewart B: Nonmuscle Myosin II helps regulate synaptic vesicle mobility at the Drosophila neuromuscular junction. BMC Neuroscience 2010, 11(1):37.

3. Gaffield MA, Betz WJ: Synaptic vesicle mobility in mouse motor nerve terminals with and without synapsin. J Neurosci 2007, 27(50):13691-13700.

4. Gaffield MA, Rizzoli SO, Betz WJ: Mobility of Synaptic Vesicles in Different Pools in Resting and Stimulated Frog Motor Nerve Terminals. Neuron 2006, 51(3):317-325.

5. Jordan R, Lemke EA, Klingauf J: Visualization of synaptic vesicle movement in intact synaptic boutons using fluorescence fluctuation spectroscopy. Biophys J 2005, 89(3):2091-2102.

6. Ryan TA: Inhibitors of myosin light chain kinase block synaptic vesicle pool mobilization during action potential firing. J Neurosci 1999, 19(4):1317-1323.

7. Tokuoka H, Goda Y: Myosin light chain kinase is not a regulator of synaptic vesicle trafficking during repetitive exocytosis in cultured hippocampal neurons. J Neurosci 2006, 26(45):11606-11614.

8. Menetrey J, Llinas P, Mukherjea M, Sweeney HL, Houdusse A: The structural basis for the large powerstroke of myosin VI. Cell 2007, 131(2):300-308.

9. Iwaki M, Tanaka H, Iwane AH, Katayama E, Ikebe M, Yanagida T: Cargobinding makes a wild-type single-headed myosin- $\mathrm{VI}$ move processively. Biophys J 2006, 90(10):3643-3652.

10. Naccache $S N$, Hasson T: Myosin VI altered at threonine 406 stabilizes actin filaments in vivo. Cell Motil Cytoskeleton 2006, 63(10):633-645.

11. Petritsch C, Tavosanis G, Turck CW, Jan LY, Jan YN: The Drosophila myosin $\mathrm{VI}$ Jaguar is required for basal protein targeting and correct spindle orientation in mitotic neuroblasts. Dev Cell 2003, 4(2):273-281.

12. Rock RS, Rice SE, Wells AL, Purcell TJ, Spudich JA, Sweeney HL: Myosin VI is a processive motor with a large step size. Proc Natl Acad Sci USA 2001, 98(24):13655-13659.

13. Hertzano R, Shalit E, Rzadzinska AK, Dror AA, Song L, Ron U, Tan JT, Shitrit AS, Fuchs $H$, Hasson T, Ben-Tal N, Sweeney HL, de Angelis MH, Steel KP, Avraham KB: A Myo6 mutation destroys coordination between the myosin heads, revealing new functions of myosin $\mathrm{VI}$ in the stereocilia of mammalian inner ear hair cells. PLOS Genet 2008, 4(10): e1000207.

14. Hicks JL, Deng WM, Rogat AD, Miller KG, Bownes M: Class VI unconventional myosin is required for spermatogenesis in Drosophila. Mol Biol Cell 1999, 10(12):4341-4353.

15. Noguchi T, Lenartowska M, Miller KG: Myosin VI stabilizes an actin network during Drosophila spermatid individualization. Mol Biol Cell 2006 17(6):2559-2571.

16. Noguchi T, Frank DJ, Isaji M, Miller KG: Coiled-coil-mediated dimerization is not required for myosin VI to stabilize actin during spermatid individualization in Drosophila melanogaster. Mol Biol Cell 2009, 20(1):358-367.

17. Geisbrecht ER, Montell DJ: Myosin VI is required for E-cadherin-mediated border cell migration. Nat Cell Biol 2002, 4(8):616-620.
18. Yoshida H, Cheng W, Hung J, Montell D, Geisbrecht E, Rosen D, Liu J, Naora $\mathrm{H}$ : Lessons from border cell migration in the Drosophila ovary: A role for myosin VI in dissemination of human ovarian cancer. Proc Natl Acad Sci USA 2004, 101(21):8144-8149.

19. Vreugde S, Ferrai C, Miluzio A, Hauben E, Marchisio PC, Crippa MP, Bussi M, Biffo S: Nuclear myosin VI enhances RNA polymerase II-dependent transcription. Mol Cell 2006, 23(5):749-755.

20. Millo H, Leaper K, Lazou V, Bownes M: Myosin VI plays a role in cell-cell adhesion during epithelial morphogenesis. Mech Dev 2004 121(11):1335-1351.

21. Maddugoda MP, Crampton MS, Shewan AM, Yap AS: Myosin VI and vinculin cooperate during the morphogenesis of cadherin cell cell contacts in mammalian epithelial cells. J Cell Biol 2007, 178(3):529-540.

22. Sahlender DA, Roberts RC, Arden SD, Spudich G, Taylor MJ, Luzio JP, Kendrick-Jones J, Buss F: Optineurin links myosin VI to the Golgi complex and is involved in Golgi organization and exocytosis. J Cell Biol 2005, 169(2):285-295.

23. Bond LM, Peden AA, Kendrick-Jones J, Sellers JR, Buss F: Myosin VI and its binding partner optineurin are involved in secretory vesicle fusion at the plasma membrane. Mol Biol Cell 2011, 22(1):54-65.

24. Buss F, Arden SD, Lindsay M, Luzio JP, Kendrick-Jones J: Myosin VI isoform localized to clathrin-coated vesicles with a role in clathrin-mediated endocytosis. EMBO J 2001, 20(14):3676-3684.

25. Aschenbrenner $L$, Naccache SN, Hasson T: Uncoated endocytic vesicles require the unconventional myosin, Myo6, for rapid transport through actin barriers. Mol Biol Cell 2004, 15(5):2253-2263.

26. Puri C: Loss of myosin VI no insert isoform (Nol) induces a defect in clathrin-mediated endocytosis and leads to caveolar endocytosis of transferrin receptor. J Biol Chem 2009, 284(50):34998-35014.

27. Park H, Ramamurthy B, Travaglia M, Safer D, Chen LQ, Franzini-Armstrong C, Selvin PR, Sweeney HL: Full-length myosin VI dimerizes and moves processively along actin filaments upon monomer clustering. $\mathrm{Mol} \mathrm{Cell}$ 2006, 21(3):331-336.

28. Osterweil E, Wells DG, Mooseker MS: A role for myosin VI in postsynaptic structure and glutamate receptor endocytosis. J Cell Biol 2005, 168(2):329-338.

29. Yano $H$, Ninan I, Zhang H, Milner TA, Arancio O, Chao MV: BDNF-mediated neurotransmission relies upon a myosin VI motor complex. Nat Neurosci 2006, 9(8):1009-1018.

30. Erben V, Waldhuber M, Langer D, Fetka I, Jansen RP, Petritsch C Asymmetric localization of the adaptor protein Miranda in neuroblasts is achieved by diffusion and sequential interaction of Myosin II and VI. J Cell Sci 2008, 121(Pt 9):1403-1414.

31. Peyre JB, Seabrooke S, Randlett O, Kisiel M, Aigaki T, Stewart BA: Interaction of cytoskeleton genes with NSF2-induced neuromuscular junction overgrowth. Genesis 2006, 44(12):595-600.

32. Morrison JK, Miller KG: Genetic characterization of the Drosophila jaguar322 mutant reveals that complete myosin VI loss of function is not lethal. Genetics 2008, 179(1):711-716.

33. Sokolowski MB, Hansell KP: The foraging locus: behavioral tests for normal muscle movement in rover and sitter Drosophila melanogaster larvae. Genetica 1992, 85(3):205-209.

34. Millo $\mathrm{H}$, Bownes M: The expression pattern and cellular localisation of Myosin VI during the Drosophila melanogaster life cycle. Gene Expr Patterns 2007, 7(4):501-510.

35. Atwood HL, Govind CK, Wu CF: Differential ultrastructure of synaptic terminals on ventral longitudinal abdominal muscles in Drosophila larvae. J Neurobiol 1993, 24(8):1008-1024.

36. Stimson DT, Estes PS, Rao S, Krishnan KS, Kelly LE, Ramaswami M: Drosophila stoned proteins regulate the rate and fidelity of synaptic vesicle internalization. J Neurosci 2001, 21(9):3034-3044.

37. Rodesch CK, Broadie K: Genetic studies in Drosophila: vesicle pools and cytoskeleton-based regulation of synaptic transmission. Neuroreport 2000, 11(18):R45-53.

38. Zucker RS: Calcium- and activity-dependent synaptic plasticity. Curr Opin Neurobiol 1999, 9(3):305-313.

39. Zucker RS, Regehr WG: Short-term synaptic plasticity. Annu Rev Physiol 2002, 64:355-405.

40. Zucker RS, Delaney KR, Mulkey R, Tank DW: Presynaptic calcium in transmitter release and posttetanic potentiation. Ann N Y Acad Sci 1991, 635:191-207 
41. Hoang B, Chiba A: Single-cell analysis of Drosophila larval neuromuscular synapses. Dev Biol 2001, 229(1):55-70

42. Zhang B: Genetic and molecular analysis of synaptic vesicle recycling in Drosophila. J Neurocytol 2003, 32(5-8):567-589.

43. Akbergenova Y, Bykhovskaia M: Synapsin maintains the reserve vesicle pool and spatial segregation of the recycling pool in Drosophila presynaptic boutons. Brain Res 2007, 1178:52-64.

44. Akbergenova Y, Bykhovskaia M: Synapsin regulates vesicle organization and activity-dependent recycling at Drosophila motor boutons. Neuroscience 2010, 170(2):441-452.

45. Pawson C, Eaton BA, Davis GW: Formin-dependent synaptic growth: evidence that Dlar signals via Diaphanous to modulate synaptic actin and dynamic pioneer microtubules. J Neurosci 2008, 28(44):11111-11123.

46. Mermall V, Miller KG: The 95F unconventional myosin is required for proper organization of the Drosophila syncytial blastoderm. J Cell Biol 1995, 129(6):1575-1588.

47. Kuromi H, Kidokoro Y: Two distinct pools of synaptic vesicles in single presynaptic boutons in a temperature-sensitive Drosophila mutant, shibire. Neuron 1998, 20(5):917-925.

48. Rizzoli SO, Betz WJ: Synaptic vesicle pools. Nat Rev Neurosci 2005, 6(1):57-69

49. Delgado R, Maureira C, Oliva C, Kidokoro Y, Labarca P: Size of vesicle pools, rates of mobilization, and recycling at neuromuscular synapses of a Drosophila mutant, shibire. Neuron 2000, 28(3):941-953.

50. Kuromi H, Kidokoro Y: Tetanic stimulation recruits vesicles from reserve pool via a cAMP-mediated process in Drosophila synapses. Neuron 2000, 27(1):133-143.

51. Stewart BA, McLean JR: Population density regulates Drosophila synaptic morphology in a Fasciclin-II-dependent manner. J Neurobiol 2004, 61(3):392-399.

52. Dickman DK, Horne JA, Meinertzhagen IA, Schwarz TL: A slowed classical pathway rather than kiss-and-run mediates endocytosis at synapses lacking synaptojanin and endophilin. Cell 2005, 123(3):521-533.

53. Koh TW, Korolchuk VI, Wairkar YP, Jiao W, Evergren E, Pan H, Zhou Y, Venken KJ, Shupliakov O, Robinson IM, O'Kane CJ, Bellen HJ: Eps15 and Dap160 control synaptic vesicle membrane retrieval and synapse development. J Cell Biol 2007, 178(2):309-322.

54. Sambrook J: Molecular cloning: a laboratory manual. 3 edition. Cold Spring Harbor, N.Y.: Cold Spring Harbor Laboratory Press; 2001

55. Pereira HS, MacDonald DE, Hilliker AJ, Sokolowski MB: Chaser (Csr), a new gene affecting larval foraging behavior in Drosophila melanogaster. Genetics 1995, 141(1):263-270.

56. Stewart BA, Mohtashami M, Rivlin P, Deitcher DL, Trimble WS, Boulianne GL Dominant-negative NSF2 disrupts the structure and function of Drosophila neuromuscular synapses. J Neurobiol 2002, 51(4):261-271.

57. Stewart BA, Atwood HL, Renger JJ, Wang J, Wu CF: Improved stability of Drosophila larval neuromuscular preparations in haemolymph-like physiological solutions. J Comp Physiol A 1994, 175(2):179-191.

58. Seabrooke S, Stewart BA: Moesin helps to restrain synaptic growth at the Drosophila neuromuscular junction. Dev Neurobiol 2008, 68(3):379-391.

59. Sullivan W, Ashburner M, Hawley RS: Drosophila protocols Cold Spring Harbor, N.Y.: Cold Spring Harbor Laboratory Press; 2000.

\section{doi:10.1186/1471-2202-12-65}

Cite this article as: Kisiel et al:: Myosin VI contributes to synaptic transmission and development at the Drosophila neuromuscular junction. BMC Neuroscience 2011 12:65.

\section{Submit your next manuscript to BioMed Central and take full advantage of:}

- Convenient online submission

- Thorough peer review

- No space constraints or color figure charges

- Immediate publication on acceptance

- Inclusion in PubMed, CAS, Scopus and Google Scholar

- Research which is freely available for redistribution

Submit your manuscript at www.biomedcentral.com/submit
Biomed Central 